\title{
Effect of strain gage misalignment on cross sensitivity of extended ring (ER) transducers
}

\author{
N.B. McLaughlin ${ }^{1 *}$ and Y. Chen ${ }^{2}$ \\ ${ }^{1}$ Research Scientist, Eastern Cereal and Oilseed Research Centre, Research Branch, Agriculture and Agri-Food \\ Canada, 960 Carling Ave., Ottawa, ON K1A 0C6 Canada \\ ${ }^{2}$ Professor, Department of Biosystems Engineering, E2-376 EITC, University of Manitoba, Winnipeg, MB R3T 2N2 \\ Canada \\ *Email:neil.mclaughlin@agr.gc.ca
}

McLaughlin, N.B., and Y. Chen. 2012. Effect of strain gage misalignment on cross sensitivity of extended ring (ER) transducers. Canadian Biosystems Engineering/Le génie des biosystèmes au Canada 54: 2.23-2.31. The extended octagonal ring (EOR) and plain extended ring (ER) transducers are popular devices for multi-dimensional force and moment measurement in agricultural engineering research. Independent measurements of forces and moments are accomplished by strain gages strategically located in ring sections at either end of the devices. Much effort has been devoted to determining the optimal location of strain gages to minimize cross sensitivity. This paper presents a theoretical analysis using analytical equations derived for the plain extended ring transducer to determine the effect of strain gage misalignment on cross sensitivity.

The analysis showed that a tangential misalignment of one degree for one strain gage in a four arm bridge would result in a cross sensitivity of approximately $0.004 \mathrm{~N} \mathrm{~N}^{-1}$ for the horizontal strain gage bridge to vertical loading, and $0.012 \mathrm{~N} \mathrm{~N}^{-1}$ for the vertical bridge to horizontal loading. Angular misalignment had little effect when the strain gages were located at the secondary strain nodes, but had a large effect when strain gages were located in regions of high strain resulting from secondary loading. Cross sensitivity was relatively independent of nominal angular location of the strain gage bridge except for a discontinuity near the primary strain nodes. The analysis demonstrates the importance of careful alignment of strain gages on extended ring transducers to minimize cross sensitivity. Key Words: Extended Octagonal Ring, Extended Ring, Cross Sensitivity, Strain Gage Misalignment.

Les dispositifs de mesure des forces de type anneau octogonal élargi (EOR) et anneau élargi (ER) sont des appareils populaires pour la mesure multidimensionnelle de force et de couple en recherche en ingénierie agricole. Des mesures indépendantes de forces et de moments sont obtenues à l'aide de jauges extensométriques placées stratégiquement dans les sections de l'anneau aux extrémités des appareils. Beaucoup d'attention a été portée à déterminer le positionnement optimal des jauges extensiométriques pour minimiser la sensibilité croisée. Cette publication décrit une analyse théorique utilisant des équations analytiques dérivées pour le dispositif de mesure de force de type anneau élargi pour déterminer l'effet du désalignement de jauges extensiométriques sur sensibilité croisée.

Les analyses ont démontré qu'un désalignement tangentiel de l'ordre de un degré pour une jauge extensiométrique dans une des quatre branches du pont pouvait entraîner une sensibilité croisée d'approximativement $0,004 \mathrm{~N} \mathrm{~N}^{-1}$ pour le pont horizontal de jauge extensiométrique pour une charge verticale et $0,012 \mathrm{~N} \mathrm{~N}^{-1}$ pour le pont vertical de jauge extensiométrique pour une charge horizontale. Un désalignement angulaire avait peu d'effet lorsque les jauges extensiométriques étaient situées aux noeuds de charge secondaire mais avait un plus grand effet lorsque les jauges extensiométriques étaient situées dans des zones de haute charge résultant de charges secondaires. La sensibilité croisée était relativement indépendante des emplacements angulaires nominaux du pont de jauges extensiométriques à l'exception d'une discontinuité près des noeuds de charge primaire. L'analyse démontre l'importance d'un alignement précis des jauges extensiométriques sur les dispositifs de mesure des forces de type anneau élargi pour minimiser la sensibilité croisée. Mots clés: dispositifs de mesure des forces de type anneau octogonal élargi, anneau élargi, sensibilité croisée, désalignement de jauge extensiométrique.

\section{INTRODUCTION}

The extended octagonal ring (EOR) is a popular device for measurement of forces and moments in agricultural engineering research. The EOR is a variation of the extended ring (ER) transducer which itself is a variation of the circular proving ring. Both the EOR and the ER have massive central sections joined at either end by a thin ring section (Figs. 1 and 2). The plane outside ring surfaces on the EOR is easier to machine than the semi-circular outside ring surfaces of the ER, and consequently, the EOR is more commonly used. The large central section offers a variety of options for attaching the transducer to loading devices. Loading causes deformation in the thin ring sections at either end, and this deformation is measured with strain gages strategically placed in regions of high tangential ring strain.

Hoag and Yoerger (1975) developed an elegant set of analytical equations to describe the distribution of bending moment and strain in the ring sections of an ER. McLaughlin (1996) noted and corrected several typographical errors in the equations. Throughout this paper, reference to the equations will be to the original authors (Hoag and Yoerger 1975) but the reader is reminded to check McLaughlin (1996) for the corrected equations. Analytical equations for strain distribution in the EOR are not available, and many researchers designing EOR's for specific applications use the Hoag and Yoerger (1975) equations developed for ER transducers to approximate the strain in an EOR transducer. 




Fig. 1. Diagram of Extended Ring (ER) with dimensions, coordinate system and sign conventions.

Load cells usually exhibit a certain amount of cross sensitivity where extraneous forces and moments orthogonal to the primary force or moment being measured result in small signals and a corresponding "apparent" load on the primary axis. These signals add to the signals from the actual load on the primary axis, and result in an incorrect measurement of primary load. Cross sensitivity in this paper is defined as the ratio of apparent load measured on the primary axis to actual load applied on the secondary axis as given in Eq. 1 .

$$
\text { Cross Sensitivity }=\frac{\text { Apparent Primary load }}{\text { Applied Secondary load }}
$$

Throughout this paper, cross sensitivity of a primary axis to a secondary load will be denoted PxS where P and $\mathrm{S}$ refer to the primary and secondary axes respectively. This notation is not to be interpreted as related to the vector cross product which has a similar notation. Most commercial load cells are designed to measure force in one direction, but this measurement is potentially affected by each of the five secondary loads, two orthogonal forces, and three orthogonal moments. Cross sensitivity is generally very small on high quality commercial load cells, but it can be substantial on "home made" load cells such as EOR's where precision facilities for design and fabrication are not available.

Cross sensitivity is often reported as a dimensionless percentage. For example, a cross sensitivity of $1 \%$ means that a $100 \mathrm{~N}$ load on a secondary axis would produce a signal on the primary axis equivalent to $1 \mathrm{~N}$ primary load. Expressing cross sensitivity, as a percent is not appropriate when one of the primary or secondary loads is a force and the other is a moment. In this paper, the units of the primary and secondary loads in Eq. 1 will be retained for all cross sensitivities.

Much effort has been devoted to determining the optimum locations for strain gages on the ring sections of an EOR to both maximize sensitivity on the primary axis, and minimize cross sensitivity from extraneous forces and moments on secondary axes. Strain gages for
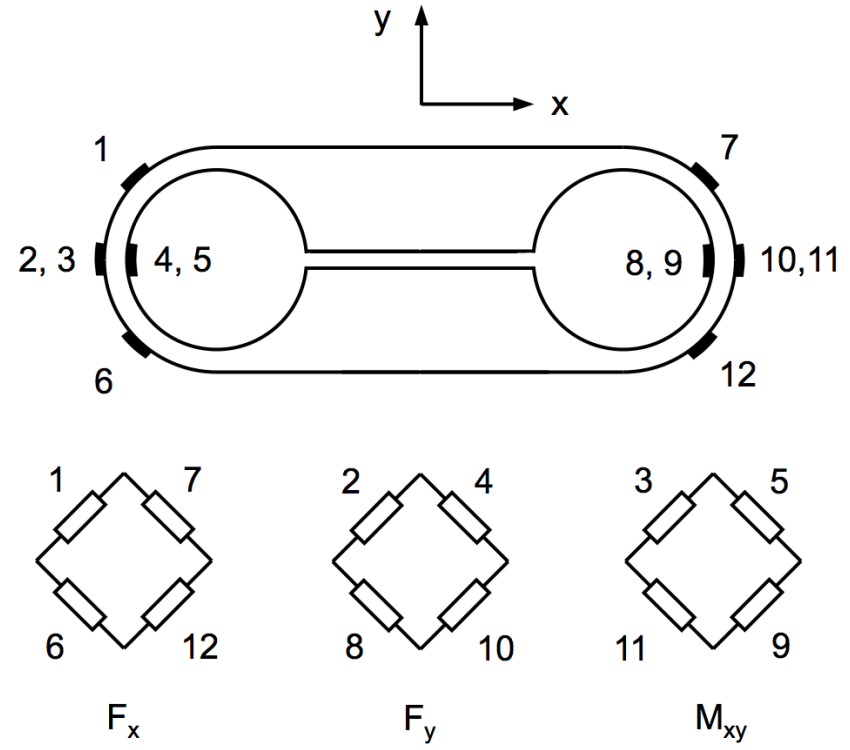

Fig. 2. Diagram of strain gage locations and bridge connection for horizontal force, vertical force and moment measurement.

measurement of the primary load component are usually placed at the strain nodes (point of zero strain) for the secondary load component to minimize cross sensitivity. The nodes for an ER can be easily determined from the Hoag and Yoerger (1975) analytical equations; many researchers have used these equations as an approximation for the EOR. Different methods have been used to examine strain distribution and locate strain nodes in an EOR including photoelastic methods (Loewen and Cook 1956; Pang et al. 1988), an array of gages at several points on the ring (Godwin 1975), and Finite Element Method (FEM), (Majumdar et al. 1994; Chen et al. 2007). There is some disagreement in the literature on optimum locations of the strain gages.

The quest for locating strain nodes seems to be based on the assumption that locating strain gages at the secondary strain nodes will result in minimum cross sensitivity. Little attention has been paid to the importance of accurately locating all four strain gages of a bridge at the same angle to maintain symmetry. Accurate alignment of gages on the inside surface of the rings is particularly difficult to achieve without specialized equipment as the rings obstruct access and visibility.

The ER and EOR have a high degree of symmetry and this attribute is the basis of their ability to isolate load components in multi-dimensional loading scenarios. However, even though the strain for vertical and horizontal loading is zero at the respective strain nodes, the strain gradient is quite high at these points. A small tangential misalignment, i.e. $\phi=\phi_{\mathrm{n}}+\Delta \phi$ (symbols are defined in List of Symbols section) for one of the four strain gages in the bridge, means that the gage is not experiencing the zero strain from the secondary load that was expected at the 

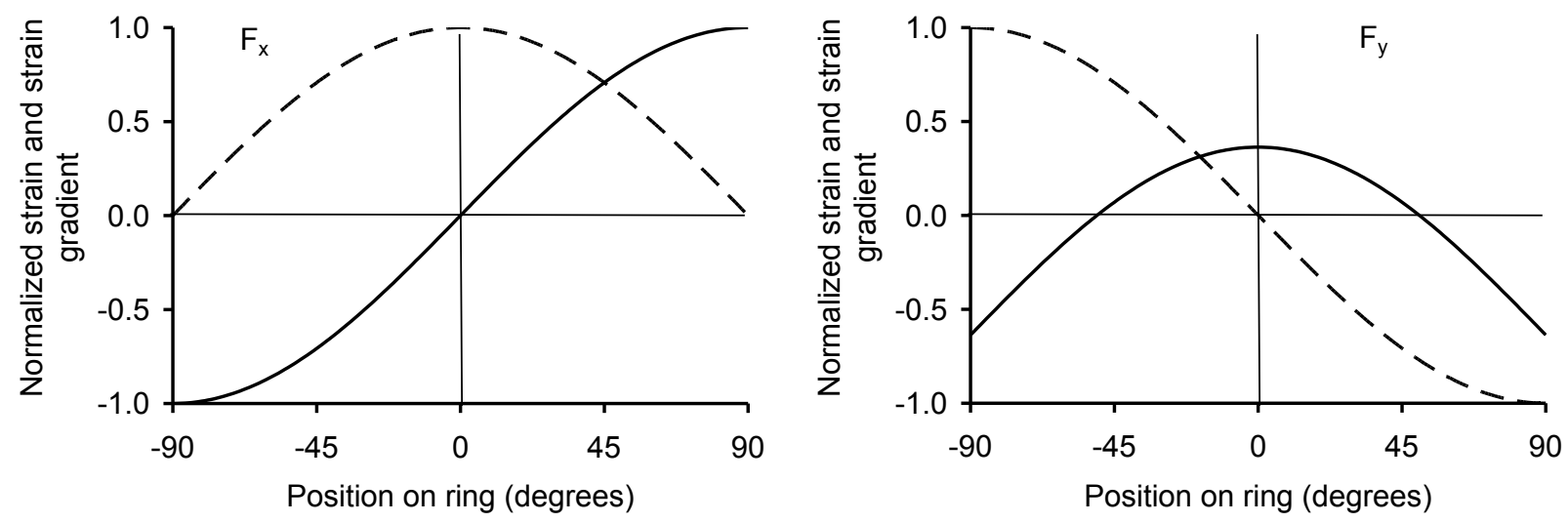

Fig. 3. Normalized strain (solid) and strain gradient (dot) distribution in right hand ring of an Extended Ring (ER) transducer for horizontal (top) and vertical (bottom) loading.

strain node. Symmetry of the bridge is violated when a gage is misaligned, and cross sensitivity can result. The objective of this paper is to quantify the effect strain gage misalignment on cross sensitivity of an extended ring (ER) transducer.

\section{ANALYTICAL EQUATIONS}

The analytical analysis is based on the Hoag and Yoerger (1975) equations for bending moment distribution in the ring sections of a plain extended ring (ER) transducer. Equations 1, 2 and 3 for ring bending moment, $M$, and strain, $\varepsilon$ have been transformed from their original form presented by Hoag and Yoerger (1975) to that for a right hand coordinate system with positive forces in the positive direction as shown in Fig. 1.

$$
M_{\phi}=-\frac{F_{x} R}{2} \sin \phi+\frac{F_{y} R}{2}\left(\cos \phi-\frac{2}{\pi}\right)-\frac{M_{x y}\left[\left(2+\frac{R \pi}{2 L}\right)-\left(\frac{2 R}{L}+\pi\right) \cos \phi\right]}{\left(8+\frac{R \pi}{L}+\frac{2 L \pi}{R}\right)}
$$

$M_{\phi}=\frac{F_{x} R}{2} \sin \phi-\frac{F_{y} R}{2}\left(\frac{2}{\pi}+\cos \phi\right)+\frac{M_{x y}\left[\left(2+\frac{R \pi}{2 L}\right)+\left(\frac{2 R}{L}+\pi\right) \cos \phi\right]}{\left(8+\frac{R \pi}{L}+\frac{2 L \pi}{R}\right)}$

$$
\varepsilon=\frac{6 M_{\phi}}{E b t^{2}}
$$

Equation 2 is for the right hand ring $\left(-90^{\circ}<\phi<90^{\circ}\right)$ while Eq. 3 is for the left hand ring $\left(90^{\circ}<\phi<270^{\circ}\right)$. The discussion from this point on will be confined to the right hand ring; equations can be derived for the left hand ring by manipulating Eq. 3 in a similar manner. The strain nodes for the ER transducer can be found by setting the ring bending moment in Eq. 1 equal to zero and solving for the angle $\phi$ for different one-dimensional loading scenarios. For horizontal and vertical forces, $F_{x}$ and $F_{y}$, the strain nodes for the right hand ring are at $\phi=0^{\circ}$ and $\phi$ $= \pm 50.5^{\circ}$ respectively, and are independent of the ER dimensions. For applied moment, $M_{x y}$, the position of the nodes depends on the ratio of the ring dimensions, $R$ and $L$, and is given by Eq. 4 for the right hand ring.

$$
\phi=\cos ^{-1}\left[\left(2+\frac{\pi R}{2 L}\right) \div\left(\frac{2 R}{L}+\pi\right)\right]
$$

The tangential strain gradient can be calculated by substituting Eq. 4 into Eq. 2 and differentiating with respect to angle $\phi$.

$$
\frac{\partial \varepsilon}{\partial \phi}=\frac{6}{E b t^{2}}\left\{-\frac{F_{x} R}{2} \cos \phi-\frac{F_{y} R}{2} \sin \phi-\frac{M_{x y}\left(\frac{2 R}{L}+\pi\right) \sin \phi}{\left(8+\frac{R \pi}{L}+\frac{2 L \pi}{R}\right)}\right\}
$$

The location of the maximum absolute strain gradient determined by setting the second derivative of Eq. 2 equal to zero and solving for $\phi$ is at $\phi=0^{\circ}$ for horizontal loading (Fig. 3) This is the exact location of the strain node for horizontal loading, the position normally selected for locating the vertical strain gages (Fig. 2). The location for maximum strain gradient for vertical loading is at $\phi= \pm 90$ ${ }^{\circ}$ and the strain gradient for vertical loading is $77 \%$ of its maximum value at the vertical strain nodes at $\pm 50.5^{\circ}$.

\section{Types of strain gage misalignment}

There are three degrees of freedom and three possible types of misalignment in locating strain gages on the ring 
sections, 1) axial, 2) tangential, and 3) angular. Each type of misalignment has a different effect on cross sensitivity.

Axial misalignment: Axial misalignment refers to the gage being located off the centre line of the ER, i.e. $z \neq 0$. When loading in only two dimensions in the $x-y$ plane is considered (i.e. $F_{x}, F_{y}$ and $M_{x y}$ ), axial misalignment in the $z$-direction has no effect on either ER sensitivity or cross sensitivity.

Tangential misalignment: Tangential misalignment means that the strain gage is not located precisely at the target angle $\phi$ in the $x-y$ plane. The ER and EOR both have a high degree of symmetry, and the ability to isolate and measure one-dimensional forces and moments from a multi-dimensional loading scenario stems from the symmetry of the devices. All gages in a bridge must be placed at the same angle on either side of the $x$-axis to achieve this symmetry.

Angular misalignment: In angular misalignment, the gage is located in the centre of the ER in the $z$-direction, i.e. on the neutral axis, and is located at the desired angle $\phi$ in the $x-y$ plane, but is rotated by an angle $\theta$ so that the gage grid is not parallel to the $x-y$ plane. Consequently, the gage has some sensitivity to Poisson strain in the $z$ direction, and is slightly less sensitive to tangential strain in the $x-y$ plane.

\section{Effect of tangential strain gage misalignment}

The bridge voltage is given by the well-known equation for a four arm strain gage bridge.

$$
V=\frac{V_{o} G F\left(\varepsilon_{1}-\varepsilon_{2}+\varepsilon_{3}-\varepsilon_{4}\right)}{4}
$$

The change in bridge voltage, $\Delta V$, due to tangential misalignment of one gage by an angle $\Delta \phi$, can be approximated with the first term of a Taylor Series expansion of Eq. 7 .

$$
\Delta V=\frac{\partial V}{\partial \phi} \Delta \phi=\frac{\partial V}{\partial \varepsilon} \frac{\partial \varepsilon}{\partial \phi} \Delta \phi
$$

The first term in Eq. 8 can be found by differentiating Eq. 7 with respect to $\varepsilon$ for the arm in the four arm bridge corresponding to the misaligned gage.

$$
\frac{\partial V}{\partial \varepsilon}=\frac{V_{0} G F}{4}
$$

Substituting Eq. 9 and 6 into Eq. 8 yields an expression for a change in bridge voltage due to tangential misalignment of $\Delta \phi$ for one of the four gages in a strain gage bridge.

$$
\Delta V=V_{o} \frac{G F}{4} \frac{6}{E b t^{2}}\left\{-\frac{F_{x} R}{2} \cos \phi-\frac{F_{y} R}{2} \sin \phi-\frac{M_{x y}\left(\frac{2 R}{L}+\pi\right) \sin \phi}{\left(8+\frac{R \pi}{L}+\frac{2 L \pi}{R}\right)}\right\} \Delta \phi
$$

It is assumed that perfect symmetry is achieved for the other three gages in the bridge, i.e. they are located at $\pm \phi$ and $180^{\circ} \pm \phi$ in the right and left hand rings respectively.

Equation 10 provides a general expression for the change in bridge voltage for any strain gage bridge with gages located symmetrically on the ring at angle $\pm \phi$, and with one gage misaligned tangentially by $\Delta \phi$ and with bridge connections for force or moment measurement as given in Fig. 2. Equation 10 reveals that when $\Delta \phi \neq 0$, a secondary load will produce a signal on the primary bridge even if the primary bridge is located at the strain node for secondary loading. This is cross sensitivity as defined by Eq. 1. Further, if $\Delta \phi=0$, symmetry is preserved and cross sensitivity will be zero for bridges with strain gages nominally located at any angle $\pm \phi$. This implies that it is not necessary to locate strain gages at the strain nodes to eliminate cross sensitivity; however, strain gages must be properly aligned to achieve this attribute.

\section{Effect of angular strain gage misalignment}

By definition, the tangential strain is uni-directional in the $x-y$ plane and is tangent to the ring surface when loading in only two dimensions is considered. However, the tangential strain in the $x-y$ plane resulting from any combination of the three load components, $F_{x}, F_{y}$ and $M_{x y}$ will always be accompanied by Poisson strain in the $z$ direction and strain gages that are misaligned angularly will have some sensitivity to Poisson strain in the $z$ direction, and slightly reduced sensitivity to tangential strain. Strain parallel to the strain gage grid for a gage oriented at an angle $\theta$ to the $x-y$ plane is given the wellknown strain transformation equation.

$$
\varepsilon_{\theta}=\frac{\varepsilon_{t}+\varepsilon_{z}}{2}+\frac{\varepsilon_{t}-\varepsilon_{z}}{2} \cos 2 \theta+\frac{\gamma_{t z}}{2} \sin 2 \theta
$$

For two-dimensional loading, the surface shear strain, $\gamma_{t z}$ is zero. The effect of angular misalignment of one gage on cross sensitivity can be determined by calculating tangential and Poisson strain, $\varepsilon_{t}$ and $\varepsilon_{z}$, at gage location $\phi$ from Eq. 2 and 4, and $\varepsilon_{\theta}$ from Eq. 11 and substituting into Eqs. 7 and 1.

\section{Effect of mismatched strain gages}

The previous analysis is based on the assumption that all gages in a bridge are perfectly matched, and have the same gage factor. If one gage has a smaller gage factor than the other three, then the change in gage resistance of the mismatched strain gage for a given strain will be smaller than that for the other three strain gages. This has the same effect on bridge output voltage as a smaller strain on the mismatched gage. Modern strain gages from the same lot have nearly identical gage factors, usually within \pm 0.5 $\%$. Strain gage manufacturers recommend that all gages in a strain gage bridge be from the same lot to minimize mismatch among the gages.

\section{Numerical simulation}

Numerical simulations were done for an ER with ring radius of $50 \mathrm{~mm}$, and $\mathrm{L} / \mathrm{R}=2.0$. A strain gage installation 
Table 1. Cross sensitivity for $E R(L / R=2)$ with three strain gages in each bridge located at the same angle, $\pm \phi$, on the ring on either side of the $x$-axis, and one gage misaligned tangentially by one degree $\left(\Delta \phi=1^{\circ}, \theta=0\right)$, angularly by one degree $\left(\Delta \phi=0, \theta=1^{\circ}\right)$, and both tangentially and angularly by one degree $\left(\Delta \phi=1^{\circ}, \theta=\right.$ $\left.\mathbf{1}^{\mathbf{0}}\right)$.

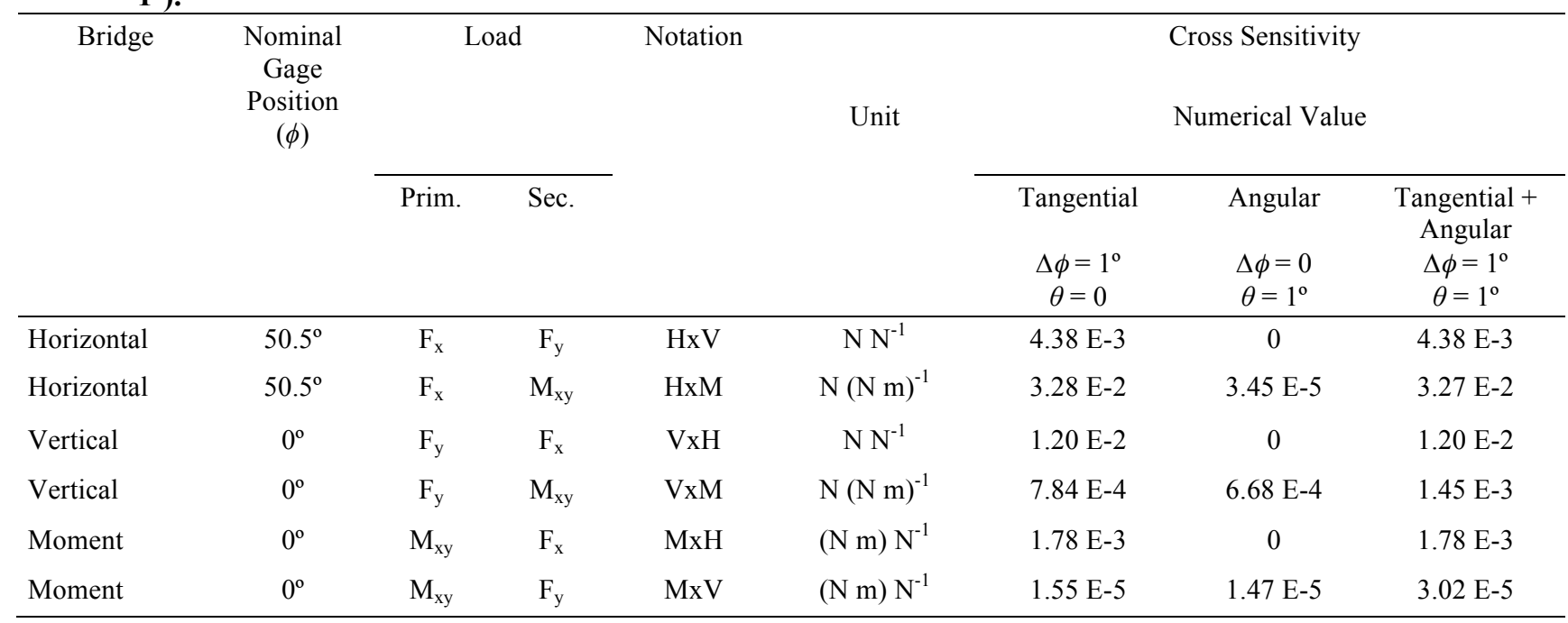

with three bridges for measurement of horizontal, vertical and moment loading was assumed with all four gages of each bridge in perfect symmetry as shown in Fig. 2. One gage in each bridge was then assigned a tangential and / or angular misalignment, and cross sensitivities were calculated using Eqs. 1, 2, 3, 4, 7 and 11. The strains, respective bridge voltages, and resulting cross sensitivities calculated from these equations were exact for the respective positions on the rings (subject to assumptions in the Hoag and Yoerger (1975) equations), and were not subject to any errors by approximations with the first term of the Taylor Series expansions in Eq. 8.

\section{RESULTS}

\section{Effect of tangential misalignment for gages nominally} located at strain nodes

Calculated cross sensitivities for strain gages nominally located at the strain nodes as shown in Fig. 2, and with one gage in each bridge with a tangential and / or angular misalignment of one degree are given in Table 1. Cross sensitivity of the horizontal bridge to vertical loading, $\mathrm{HxV}$, was $4.38 \times 10^{-3} \mathrm{~N} \mathrm{~N}^{-1}$, and cross sensitivity of the vertical bridge to horizontal loading, $\mathrm{VxH}$, was $1.20 \times 10^{-2}$ $\mathrm{N} \mathrm{N}^{-1}$, nearly three times $\mathrm{HxV}$. Sensitivity of an ER to vertical loading is much lower than sensitivity to horizontal loading (Eq. 2) giving rise to higher cross sensitivity for the vertical bridge.

Cross sensitivity of the moment bridge to vertical and horizontal loading, $\mathrm{MxV}$ and $\mathrm{MxH}$, was $1.78 \times 10^{-3}$ and $1.55 \times 10^{-5} \mathrm{~N} \mathrm{~m} \mathrm{~N}^{-1}$ respectively, and cross sensitivity of the horizontal and vertical bridges to moment loading, HxM and VxM, was $3.28 \times 10^{-2}$ and $7.84 \times 10^{-4} \mathrm{~N}(\mathrm{~N} \mathrm{~m})^{-1}$ respectively. The much smaller numerical values of the cross sensitivities of the moment bridges to secondary force loading than the force bridges to secondary moment loading arise from the dimensions of the ER relative to the base unit of meter in expressing moments; the ER ring radius used in the calculations was $50 \mathrm{~mm}$, and length to radius ratio $(\mathrm{L} / \mathrm{R})$ was 2.0 .

\section{Effect of combined angular and tangential misalignment}

Plots of cross sensitivity for combined angular and tangential misalignment are given in Fig. 4. The cross sensitivities $\mathrm{HxV}, \mathrm{VxH}$, and $\mathrm{MxH}$ all show no effect of angular misalignment for perfect tangential alignment, i.e. $\Delta \phi=0$. For these three cases, the strain gages are located at the secondary strain nodes (zero strain from secondary loading), and rotating a strain gage in a zero strain field has no effect on the bridge output. For the other three cases, $\mathrm{MxV}, \mathrm{HxM}$ and $\mathrm{VxM}$, the primary bridge is not located at the strain node for secondary loading (Fig. 2, Table 1), and secondary loading produces non-zero tangential and Poisson strain at gage location resulting in an effect from angular misalignment when $\Delta \phi=0$ (Eq. 11, Fig. 4). Depending on which gage in the bridge is misaligned, angular misalignment can either subtract from, or add to the effect of tangential misalignment on cross sensitivity. Similarly, depending on which gage is misaligned, the sign of the cross sensitivity can be either positive or negative. The sign of the cross sensitivity is omitted in Table 1, and cross sensitivity from tangential and angular misalignment is taken to be additive in Fig. 4.

Angular misalignment has a large effect for VxM and $\mathrm{MxV}$ (Fig. 4). Both vertical and moment loading produce near maximum strain and zero strain gradient at $\phi$ $=0$, the location of both vertical and moment bridges (Fig. 2). High tangential and Poisson strain results in a large effect of angular misalignment, and low strain gradient results in minimal effect of tangential misalignment (Eq. 10). In contrast, tangential misalignment dominates angular misalignment for $\mathrm{VxH}, \mathrm{HxV}, \mathrm{HxM}$ and $\mathrm{MxH}$ 

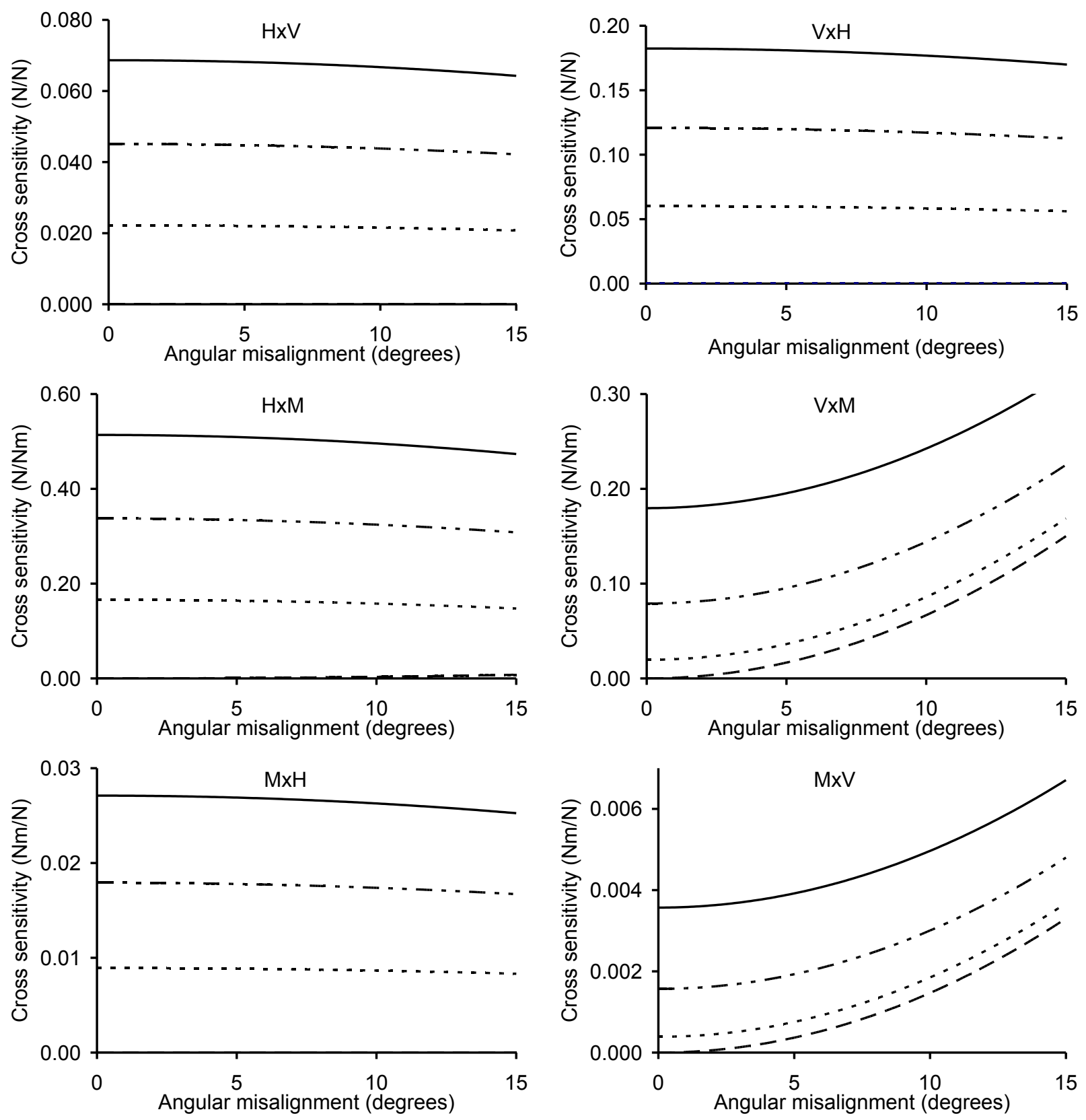

Fig. 4. Cross sensitivity among horizontal, vertical and moment bridges vs. angular misalignment of one strain gage and tangential misalignment of the same strain gage of 0 (long dash), 5 (dot), 10 (dash-dot-dot) and 15 (solid) degrees. Bridges are nominally located at positions indicated in Fig. 2. For tangential misalignment of 0 degrees, cross sensitivities $\mathrm{HxV}, \mathrm{VxH}$ and $\mathrm{MxH}$ are zero for all angular misalignments and the curves (long dash) are coincident with the $x$-axis.

(Fig. 4). For three of these cases, $\mathrm{VxH}, \mathrm{HxV}$ and $\mathrm{MxH}$, the primary gages are located at the secondary strain nodes and have zero tangential and Poisson strain, but a high strain gradient from secondary loading. These factors contribute to a small effect of angular misalignment and a large effect of tangential misalignment (Fig. 4). The horizontal bridge is located at the vertical strain node at $50.5^{\circ}$ but the moment strain node is at $47.7^{\circ}$ for $\mathrm{L} / \mathrm{R}=2$.
Secondary moment loading produces both intermediate strain and strain gradient on the horizontal bridge resulting in intermediate contributions of both tangential and angular misalignment on HxM (Table 1).

Tangential misalignment - effect of nominal gage location

Plots of cross sensitivity for one degree tangential misalignment of one gage vs. nominal gage location from 

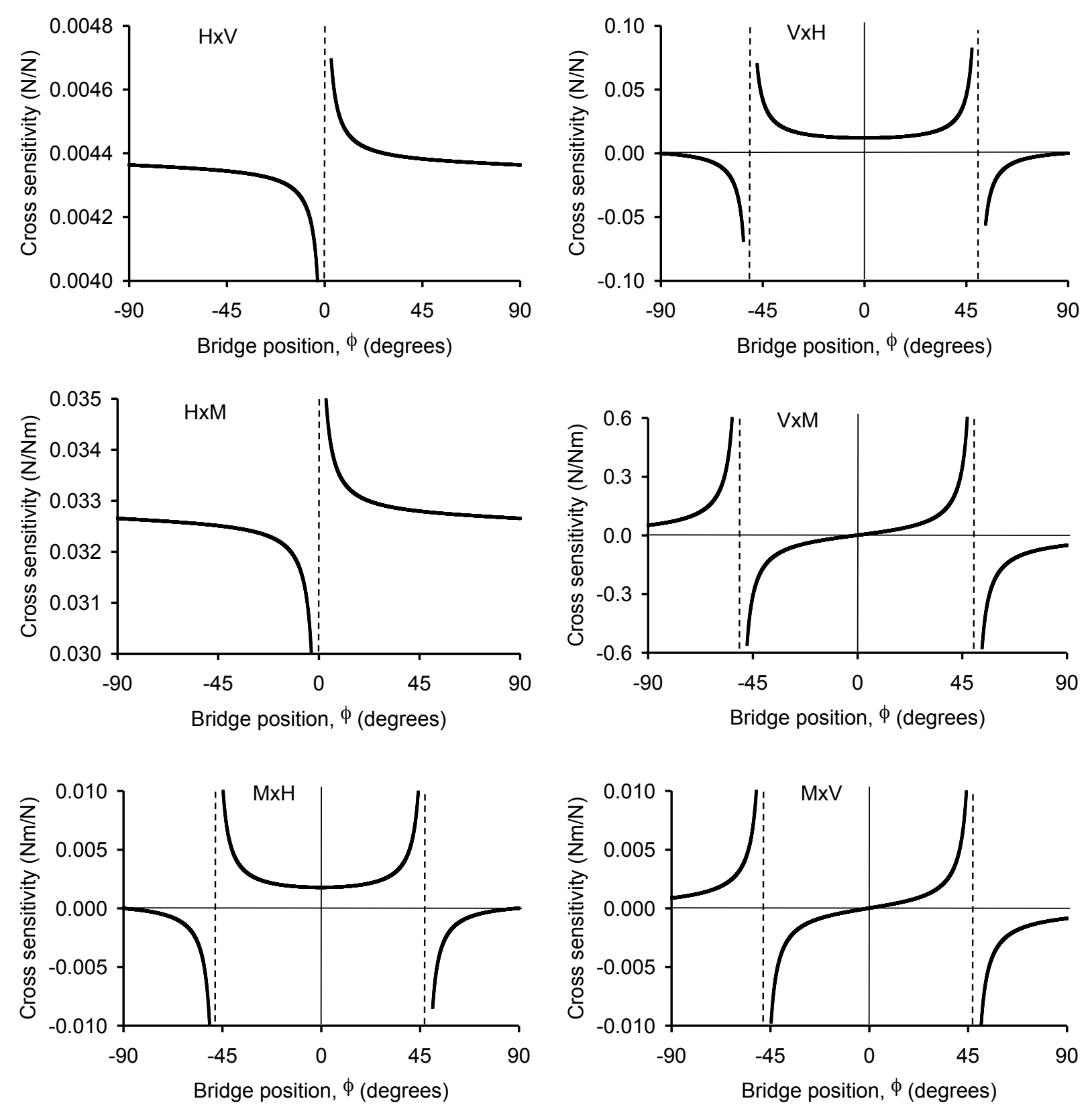

Fig. 5. Cross sensitivity between horizontal, vertical and moment bridges vs. nominal bridge position on ring to tangential misalignment of one gage by one degree. Strain nodes for primary loading and accompanying discontinuities are represented by vertical dashed lines.

$\phi=-90^{\circ}$ to $+90^{\circ}$ are given in Fig. 5 for all combinations of primary bridge and secondary loading. Each of these plots exhibits a discontinuity when the gages for the horizontal, vertical and moment bridges are nominally located at the respective primary strain nodes of $\phi=0^{\circ}, \phi= \pm 50.5^{\circ}$, and $\phi \pm 47.7^{\circ}$. The primary sensitivity is zero at the primary strain node, and any non-zero signal imposed on the primary bridge by a secondary load would be interpreted as an infinite apparent primary load therefore infinite or undefined cross sensitivity (Eq. 1). This discontinuity is of little practical significance since strain gages are normally located areas where primary sensitivity is near its maximum which are far away from the primary strain nodes.

Cross sensitivities of horizontal and vertical bridges to vertical and horizontal loading, $\mathrm{HxV}$ and $\mathrm{VxH}$ for one degree tangential misalignment was nearly constant at about 0.00438 and $0.012 \mathrm{~N} \mathrm{~N}^{-1}$ respectively over a wide range of nominal gage locations except near the respective primary nodes of $\phi=0^{\circ}$ and $\phi= \pm 50.5^{\circ}$ (Fig. 5). Similarly, cross 
sensitivities of horizontal and moment bridges to moment and horizontal loading showed similar trends but with different magnitudes. Cross sensitivities of vertical and moment bridges to moment and vertical loading were zero near their nominal location of $\phi=0^{\circ}$ with a small increase in magnitude on either side of this location. As discussed previously, the strain gradient for both vertical and moment loading is zero at $\phi=0^{\circ}$, and consequently the effect of tangential misalignment is minimal near $\phi=0^{\circ}$. However, strain is a maximum at $\phi=0^{\circ}$ for both vertical and moment loading (Fig. 3), and effect of angular misalignment would be highest at this location (Fig. 4).

The plots in Fig. 5 demonstrate that locating strain gages at the secondary strain nodes does not guarantee zero cross sensitivity. Taken together, Figs. 3, 4 and 5 suggest that the nominal location of the strain gage bridges relative to the secondary strain nodes is relatively unimportant, but careful attention to alignment to maintain symmetry is important to minimize cross sensitivity.

\section{DISCUSSION}

The analysis is based on strain at a single point on the ring sections. In reality, strain gages integrate strain over the area encompassed by the grid. The strain gradient varies throughout the ring section (Eq. 5, Fig. 3) and the integration of strain over the length of the gage grid will result in a measured strain slightly different from the strain at the gage centroid. The analysis could be readily extended to consider the effect of the length of the strain gage grid.

The analysis was for misalignment of only one gage of the four gages in a full bridge. If two or more gages were misaligned, the effect of misalignment of additional gages could either add to, or cancel the effect of misalignment of one gage, depending in which gages were misaligned, and in which direction. Similarly, the direction of cross sensitivity depends on which one of the four gages in the bridge was misaligned. The graphs in Fig. 4 were all shown as positive cross sensitivity; negative cross sensitivity could result if the misalignment was assigned to a different gage in the bridge or in a different direction.

The magnitude of strain gage misalignment is highly dependent on the skill of the installer, but even with a high level of skill, some misalignment is inevitable. Gu et al. (1993) reported cross sensitivity of an EOR of up to $12 \%$ for the sensitivity of the vertical bridge to horizontal loading, and up to $5 \%$ for sensitivity of the horizontal bridge to vertical loading. The higher cross sensitivity of the vertical bridge is consistent with the present analysis suggesting that tangential strain gage misalignment might be the underlying cause of the observed cross sensitivity. The present analysis indicates that misalignment of a single gage would have to be severe (i.e. about $10^{\circ}$ ) to cause a cross sensitivity of $12 \%$. However, if more than one gage in the bridge was misaligned, and the direction of misalignment was such that the effects were additive, it is conceivable that a moderate misalignment could yield a $12 \%$ cross sensitivity. Other factors such as machining errors resulting in slight nonsymmetry in ER or EOR dimensions could also contribute to the measured cross sensitivity. For example, evaluation of Eq. 4 shows that a dimensional difference of only 0.1 $\mathrm{mm}$ on a ring with nominal thickness of $10 \mathrm{~mm}$ results in approximately $2 \%$ difference in tangential strain at the surface.

The analyses presented here are based on analytical equations derived for the ER transducer with constant ring thickness. Analytical equations are not available for the EOR transducer, which has varying ring thickness due to the circular inner surface, and plane outer surface of the rings. Although there will undoubtedly be differences in the numerical values of cross sensitivity between the EOR and ER, it is anticipated that the EOR will exhibit the same general trends. Analyses with numerical techniques such as Finite Element Method (FEM) are required to quantify the effect of strain gage misalignment on cross sensitivity of the EOR.

Some cross sensitivity is inevitable and the magnitude of cross sensitivity for a particular device must be measured experimentally with multidimensional calibration. $\mathrm{Gu}$ et al. (1993) and McLaughlin et al. (1998) discuss compensation methods based on multiple regression analysis of calibration data.

\section{CONCLUSIONS}

Analytical equations for the distribution of strain in the ring sections of a plain extended ring (ER) transducer were used to calculate the effect of tangential and angular strain gage misalignment on cross sensitivity of an extended ring transducer. Cross sensitivity was defined as the ratio of measured apparent primary load to applied secondary load.

Cross sensitivities were calculated for various loading scenarios on an ER with four arm strain gage bridges located at the strain nodes, and one gage assigned a small tangential and/or angular misalignment. Tangential misalignment of one strain gage by one degree produced cross sensitivities of $4.38 \times 10^{-3} \mathrm{~N} \mathrm{~N}^{-1}$ and $1.2 \times 10^{-2} \mathrm{~N} \mathrm{~N}^{-1}$ for horizontal bridge to vertical loading and vertical bridge to horizontal loading respectively. Tangential misalignment had a larger effect on cross sensitivity than angular misalignment in areas of low strain and high strain gradient, but angular misalignment had a larger effect when strain gages were located in areas of high strain and low strain gradient.

The results demonstrate that locating strain gage bridges at the strain nodes does not guarantee zero cross sensitivity. Careful attention must be paid to accurate strain gage alignment to ensure symmetry and to capitalize on the theoretical ability of an ER to achieve independent measurement of horizontal and vertical forces, and applied moment under multi-dimensional loading.

\section{LIST OF SYMBOLS}

E $\quad$ ER material modulus of elasticity $(\mathrm{Pa})$

ER Abbreviation for Extended Ring

EOR Abbreviation for Extended Octagonal Ring 


\begin{tabular}{|c|c|}
\hline$F_{\mathrm{x}}$ & Applied horizontal force $(\mathrm{N})$ \\
\hline$F_{\mathrm{y}}$ & Applied vertical force $(\mathrm{N})$ \\
\hline$G F$ & $\begin{array}{l}\text { Gage Factor for strain gage }\left(G F=\Delta R R^{-1} \varepsilon^{-1}\right. \\
\text { where } R \text { is strain gage resistance) }\end{array}$ \\
\hline$L$ & $\begin{array}{l}\text { One half of distance between ER or EOR ring } \\
\text { centres }(\mathrm{m})\end{array}$ \\
\hline$M_{\mathrm{xy}}$ & Applied moment in $x-y$ plane $(\mathrm{N} \mathrm{m})$ \\
\hline$M$ & Ring bending moment at angle $\phi(\mathrm{N} \mathrm{m})$ \\
\hline PxS & $\begin{array}{l}\text { Cross sensitivity of secondary loading }(\mathrm{S}) \text { on } \\
\text { the bridge measuring the primary (P) load } \\
\text { expressed as the ratio of apparent primary } \\
\text { load to applied secondary load. }\end{array}$ \\
\hline$R$ & ER ring radius $(m)$ \\
\hline$t$ & ER ring thickness $(\mathrm{m})$ \\
\hline$V$ & Strain gage bridge signal voltage (V) \\
\hline$V_{0}$ & Strain gage bridge excitation voltage $(\mathrm{V})$ \\
\hline$w$ & ER ring width (m) \\
\hline$x, y, z$ & $\begin{array}{l}\text { Right hand coordinate system, } x \text { horizontal, } y \\
\text { vertical, } z \text { perpendicular to page (Fig. } 1 \text { ) }\end{array}$ \\
\hline$\gamma_{\mathrm{tz}}$ & $\begin{array}{l}\text { Shear strain in a plane tangent to the ring and } \\
\text { perpendicular to the } x \text {-y plane (radians) }\end{array}$ \\
\hline$\Delta \phi$ & $\begin{array}{l}\text { Tangential misalignment of one gage in a } \\
\text { bridge (deg.) }\end{array}$ \\
\hline$\varepsilon$ & Tangential axial strain $\left(\mathrm{m} \mathrm{m}^{-1}\right)$ \\
\hline$\varepsilon_{\mathrm{i}}$ & $\begin{array}{l}\text { Tangential axial strain for gage } i \text { in a bridge } \\
\left(\mathrm{m} \mathrm{m}^{-1}\right)\end{array}$ \\
\hline$\phi$ & $\begin{array}{l}\text { Angle on ring measured from the } x \text {-axis } \\
\text { (deg.) }\end{array}$ \\
\hline$\phi_{\mathrm{n}}$ & $\begin{array}{l}\text { Angle for nominal location of ring strain } \\
\text { gages (deg.) }\end{array}$ \\
\hline$\theta$ & $\begin{array}{l}\text { Angle between strain gage grid and } x-y \text { plane } \\
\text { (deg.) }\end{array}$ \\
\hline
\end{tabular}

\section{REFERENCES}

Chen, Y., N.B. McLaughlin and S. Tessier. 2007. Double extended octagonal ring (DEOR) drawbar dynamometer. Soil \& Tillage Research. 93:462-471

Godwin, R.J. 1975. An extended octagonal ring transducer for use in tillage studies. Journal of Agricultural Engineering Research. 20: 347-352.

Gu, Y., R.L. Kushwaha and G.C. Zoerb. 1993. Crosssensitivity analysis of extended octagonal ring transducer. Transactions of the ASAE. 36(6): 19671972.

Hoag, D.L. and R.R. Yoerger. 1975. Design and analysis of load rings. Transactions of the ASAE. 19(4): 9951000.

Loewen, E.G. and N.H. Cook. 1956. Metal cutting measurements and their interpretation. In eds. Mahlmann, C.V. and W.M. Murray. Proceedings of the Society for Experimental Stress Analysis. 2(3): 57-62.

Majumdar, S., E.V. Thomas and D.S. Jayas. 1994. Optimization of parameters in the design of an extended octagonal-ring transducer. Agricultural Engineering Journal. 3(4): 152-165.

McLaughlin, N.B. 1996. Correction of an error in equations for extended ring transducers. Transactions of the ASAE. 39(2): 443-444.

McLaughlin, N.B., S. Tessier and A. Guilbert. 1998. Improved double extended octagonal ring transducer for 3-D force measurement. Canadian Agricultural Engineering. 40(4): 257-264.

Pang, S.N., G.C. Zoerb and B.A. Rostad. 1988. Application of photoelastic analysis of an extended octagonal ring. ASAE/CSAE Paper \#NCR 88-105. St. Joseph, MI: ASAE. 